\title{
El método biográfico y los estudios de recepción mediática y participación virtual
}

\section{SALOMÉ SOLA-MORALES, UNIVERSIDAD DE SANTIAGO DE CHILE}

Recibido: setiembre de 2014

Aceptado: setiembre de 2014

sentido clásico, sino también sobre las nuevas formas de participación virtual. Estas vías de comunicación en entornos virtuales traen consigo nuevos interrogantes en la forma de comportarse y actuar de los individuos y los grupos. Por eso, es muy relevante utilizar herramientas complementarias que nos permitan profundizar en los procesos psico-sociales que se dan en el ámbito comunicativo.

PALABRAS CLAVE: método biográfico; historias de vida; comunicación; recepción; participación virtual.

\section{ABSTRACT}

Reception studies have traditionally focused on the scope and influence of the media on individuals and groups, using different approaches and methodologies. This article discusses the advantages, limitations and possibilities that the biographical method provides not only in research on reception (understood in a classical sense) but also in the new forms of virtual participation. These forms of communication in virtual environments bring new questions on how individuals and groups behave, think or act. Thus, it is very important to use complementary tools that allow us to delve into the psycho-social processes that occur in the communication field.

KEYWORDS: biographical method; life stories; communication; reception; virtual participation. 


\section{INTRODUCCIÓN}

En Dos estudios sobre hermenéutica Wilhelm Dilthey expresó una diferencia fundamental: las ciencias de la naturaleza explican, las del espíritu, es decir, las humanidades ylas ciencias sociales, comprenden. Así, mientras quela explicación implica que se entiendelo individual bajo lo universal, la comprensión nos permite reconocer un evento en su irreductible singularidad (2000, 103). Bajo esta premisa, al aproximarnos a la comprensión de fenómenos tales como la recepción mediática (sea recepción entendida en un sentido clásico) o la participación virtual u on-line, consideramos clave utilizar metodologías que nos permitan adentrarnos no solo en la realidad socio-histórica delos procesos, sino también en la singularidad de los entes participantes.

En el presente artículo teórico-metodológico se reflexiona y argumenta acerca de las ventajas que el método biográfico tiene en los estudios acerca de los efectos mediáticos. La tesis que se sostiene es que el uso de esta metodología puede resultar de gran utilidad a la hora de abordar procesos comunicativos de alcance psico-social, tales como la identificación con personajes o narrativas mediáticas; el fenómeno fandom, la imitación o la suplantación de identidad; la reproducción-asunción de estereotipos, de roles o actitudes; la transmisión de valores o creencias compartidas; la creación de comunidades o grupos de pertenencia; la manipulación, el engaño o la creación de avatares, etc.

Con el fin de mostrar la cantidad de posibilidades que este método puede aportar a los estudios de comunicación, y más concretamente a la investigación sobre recepción y participación virtual, se indicarán las ventajas y los puntos fuertes que este enfoque tiene, así como se indicarán algunas de sus limitaciones. En primer lugar, se realizará una retrospectiva que permita situar los orígenes del uso de las historias de vida a la luz de diversas disciplinas. En segundo lugar, se manifestará el lugar que el investigador puede desempeñar en este tipo de metodologías, adoptando una actitud cautelosa y no etnocentrista. En tercer lugar, se clarificará el uso del término de historia de vida, diferenciándolo de otros como biograma o entrevista en profundidad. En cuarto, se indicarán las ventajas y limitaciones que este enfoque puede aportar y las técnicas más recomendables para evitar sesgo o falta de representatividad en las investigaciones que lo utilicen. En quinto y último lugar, se indicarán los principales aportes e investigaciones aplicadas desde los estudios de comunicación, y más concretamente en el estudio de los efectos mediáticos sobre los individuos y grupos.

\section{ORÍGENES DEL MÉTODO}

El uso de relatos personales como método de investigación y reflexión por parte de historiadores y antropólogos, pero también y, principalmente, por parte de sociólogos, psicólogos y comunicólogos tiene una larga tradición. Como señalan Georg Misch (1951) y Paul Thompson (1988), el método biográ- 
fico nace con fines científicos con los trabajos de los primeros historiadores y sociólogos, y se remonta a finales del siglo XIX con la obra de Rufus Anderson, titulada Memoir of Catherine Brown a Christian Indian of the Cherokee Nation (1825) y, sobre todo, con los textos de Fréderic Le Play (1855) o Charles Booth (1892-1902).

Además, tuvo su aparición, como ha subrayado Jan Szczepanski, en un momento significativo: "Cuando los sociólogos renunciaron a la creación de grandes síntesis que explican en su conjunto la naturaleza de la sociedad humana y las leyes generales de su desarrollo y procedieron a la exploración empírica de cada una de las zonas de la vida social" (Szczepanski 1978, 231). Una de las primeras investigaciones que evidenciaron esta tendencia sociológica, que transitó de la mera descripción de hechos a la comprobación de hipótesis y teorías, y quizás por ello se dice que fue el verdadero punto de inflexión del método, fue la crónica de éxodos sobre el campesinado polaco de William L. Thomas y Florian Znaniecki (2004).

La trascendencia y singularidad de esta obra respecto a las anteriores se debe a su concepción ontológica de la realidad y a su énfasis en la conexión sistémica entre lo social y lo individual. Paralos investigadores, "la realidad que el sociólogo ha de investigar se compone igual de valores culturales objetivos y de valoraciones subjetivas (actitudes) de la persona individual" (Szczepanski 1978, 244). De hecho, para que los objetos naturales se conviertan en culturales, las personalidades individuales han de otorgarles, con sus actos subjetivos significado para las finalidades humanas.

La escuela sociológica de Chicago centró su foco de análisis en temáticas marginales urbanas, y así los primeros colonizados fueron sustituidos por los nuevos otros, dominados o excluidos socialmente, tales como los sin-techo, los delincuentes, los pobres, las mujeres trabajadoras, los drogadictos, los inmigrantes... (Prat 2007, 213-236). Entre algunas de las piezas más significativas, herederas de la tradición crítica de Chicago podemos hallar las siguientes: The Hobo, the Sociology os the Homeless Man, de Nels Anderson (1923); The Jack Roller. A Delinquent Boy's Own Story, de Clifford Shaw (1930); el famoso relato cruzado de Oscar Lewis, Los hijos de Sánchez (1961); La canción de Rachel, de Miguel Barnet (1979); Tante Suzanne, de Maurizio Catani, (1982); A tumba abierta. Autobiografía de un grifota, de Oriol Romaní (1983); La prostitución popular, de Pere Negre (1988) e Inmigrantes en España: vidas y experiencias, de Eugenia Ramírez (1996), entre muchas otras.

Pero el método biográfico no solo ha sido utilizado, únicamente, desde la antropología o la sociología, sino también ha sido empleado por numerosos psicólogos. Recordemos los primeros e influyentes trabajos de Charlotte Buhler (1930), por ejemplo. Y es que con razón ha sido asemejado al método psicoanalítico, ya que no podemos olvidar quelas historias de vida y, en general, todas las entrevistas medianamente serias tienen como base implícita el dialo- 
gismo bajtiano. De hecho, una historia de vida es un diálogo lleno de matices y detalles entre dos subjetividades: un observador y un observado (investigador e informante), entre un informante y sus recuerdos (reencuentro con su pasado y su memoria) y entre un informante y su entorno social (una vez publicada la historia de vida pasa a ser conocida por la sociedad).

Tampoco podemos olvidar que la historia de vida favorece la capacidad heteroglósica humana (Bajtín, 1975) de hacer hablar a través de diversas voces no solo del pasado propio, sino del pasado cultural o social del entorno o del grupo de pertenencia del informante.

Ya desde finales de los treinta, desde una perspectiva psicológica, se desarrollaron los llamados "estudios de vida" que trataban de comprender y analizar problemas concretos a través de entrevistas a muestras pequeñas de sujetos. Algunos años después, Gordon W. Allport en The Use of Personal Documents in Psychological Science (1942) analizaba las perspectivas nemotécnicas e ideográficas en profundidad.

\section{LA POSICIÓN DEL OBSERVADORY LA RELACIÓN SUJETO-OBJETO}

El método biográfico forma parte de un proceso interactivo, ya que es provocado y construido por el investigador. Por eso, no podemos olvidar que la cuestión de la interpretación sobre los otros a partir de las percepciones propias ha planteado grandes pugnas metodológicas entre autores relativistas, que ponen en duda si un observador puede captar completamente aquello que observa, y los etnólogos y antropólogos que -como nosotros- apoyan la investigación co-participante.

Si bien es cierto que mediante la observación y la interpretación de la conducta de sujetos aislados no podremos demostrar o generalizar de manera unívoca, quizás sea pertinente aclarar que mediante el uso de este método no pretendemos llegar a una única realidad objetiva y última. Por el contrario, se pretende aportar o profundizar acerca de un fenómeno subjetivo a través de marcos de interpretación que estimamos significativos. En este sentido, consideramos relevante tomar en consideración no solo la perspectiva del observador sino también la del sujeto observado, ya que ambos en el encuentro 'cara a cara' forman parte del mismo proceso.

La tradición antropológica imperialista tendió las bases de una observación unidireccional del otro, entendido como objeto de estudio exótico ${ }^{1}$ sobre el que el investigador podía emitir juicios o definiciones. Elotro, objeto definido por un ente 'superior' que es el sujeto investigador, aparece de este modo rele-

1 Claude Lévi-Strauss escribió un artículo en el que se cuestiona que pasará con la Antropología tras la descolonización. Marc Augéllamó a este momento clavela muerte del exotismo. Los autores se refieren al cambio de paradigma en el objeto de la Antropología y se cuestionan qué pasará tras la descolonización: ¿ Si no hay colonizados a quién se estudiará? 
gado al campo de la observación en sí. En ocasiones, incluso, la antropología exótica construía historias sin jamás haber visto a los informantes o haber observado los sistemas comunicativos de los mismos. Tras la descolonización este panorama cambió radicalmente en el sentido de que el sujeto y el objeto pasaron a constituir una realidad que construyen en su propia interacción. Por ello, quisiéramos aclarar que nos sentimos deudores de las aportaciones de la moderna antropología y otorgamos gran relevancia a la relación que el investigador entabla con el informante y su entorno. Es decir, consideramos que observador y observado son entes copartícipes de la investigación.

Por eso, es muy significativo como el etnólogo y los sujetos de observación comparten miradas recíprocas, ya que se convierten en representaciones los unos para los otros: tanto el etnólogo para el otro, como el otro para el etnólogo, son identificaciones en función del lugar que ocupen, de la distancia que se les otorgue. Y es cierto que la labor del observador conlleva un modo múltiple de entender y vivir la realidad propia y la ajena. La disolución del sujeto y su relativa fusión con el objeto puede ser una de las contrapartidas de la investigación basada en discursos sobre el yo o la identidad personal. Y es que acotar la idea delo que uno representa y simplificarlo mediante el lenguaje para quelos demás lo entiendan puede resultar fácilmente tendencioso.

Como es evidente, el método biográfico plantea una serie de dificultades que hay que considerar siempre a la hora de embarcarse en una investigación de estas características. En primer lugar, encontrar buenos informantes o buenos relatos que aporten elementos significativos para nuestro objeto de estudio no siempre es fácil, por lo que gran parte de la etapa inicial habrá de dedicarse a la búsqueda de informantes dispuestos a participar, y dotados de buenas historias. En segundo lugar, el excesivo control de la información por parte del investigador puede a veces entorpecer la recogida de datos. Y en tercer lugar, si dirigimos demasiado los encuentros los informantes pueden ofrecernos una información sesgada y carente de validez argumental. Conscientes de estas dificultades, lo mejor es adecuar los criterios de validezalos objetivos temáticos de la investigación, es decir, desarrollar detenidamente la fase de selección de los relatos y así poder primar la calidad sobre la cantidad.

\section{APROXIMACIONES AL TÉRMINO DE HISTORIA DE VIDA}

La noción life history (narración biográfica) comenzó a utilizarse, según G. Duncan Mitchel (1988), tras la aparición de la obra de William L. Thomas y Florian Znaniecki, The Polish Peasant in Europe and America, en 1825. Este término hacía alusión a la recolección de la narrativa de una persona por un investigador y su posterior elaboración. Además se incluía el conjunto de registros documentales y entrevistas a personas del entorno social del informante que permitieran completar y validar el relato inicial. No fue hasta años más 
tarde que apareció la noción de life story o historia de vida, que se utilizaba para referir un tipo de relato personal que podía ser publicado sin retoques ni elaboraciones previas, conservando las particularidades lingüísticas y mayor fuerza testimonial (Mitchel 1988, 247-248). Sin entretenernos en la variada y difusa delimitación terminológica acerca del término, sin duda, preferimos la definición propuesta por Juan José Pujadas en El método biográfico. Para él, la historia de vida es un estudio de caso de una persona dada, comprendiendo su relato de vida o cualquier otro tipo de información o documentación adicional que permita la reconstrucción de la biografía de la forma más exhaustiva y objetiva posible (Pujadas 1992, 13).

Quizás sea preciso delimitar teóricamente a qué nos referimos con historia de vida, ya que hay diversidad de términos similares como relato de vida o biograma, que si bien son similares no serán los utilizados en esta ocasión. Entendemos el concepto historia de vida como lo define Pujadas (1992, 13): un "estudio de caso de una persona dada, comprendiendo su relato de vida y cualquier otro tipo de información o documentación adicional que permita la reconstrucción de la biografía de la forma más exhaustiva y objetiva posible".

Mediante las realización de las historias de vida es posible aproximarse al estudio de las "lecturas" que las personas hacen de las narrativas mediáticas y el sentido que dan a éstas. Además es posible también explorar en la praxis la influencia que tienen estas representaciones mediáticas en los procesos de identificación y exclusión realizados por los individuos o los grupos. También se podría, sin duda, profundizar en las maneras de socializar respecto a los medios, que se dan en la recepción mediática o participación virtual. Como señala Luciano H. Elizalde $(1998,17)$, "se consideran "relaciones sociales con los medios" a todo tipo de contacto o conexión -espacial, temporal, social, cultural, económico, psicológico, corporal- que se realizan entre los medios y las personas." Tampoco podemos olvidar que muchas de las relaciones que una persona establece con otras, al igual que muchas de las identificaciones subjetivas realizadas no tienen por qué ser siempre intenciones conscientes. Además, las referencias al "mundo" -social, cultural, subjetivo, material, etc.- que el hablante realiza conjugan dialécticamente actos y palabras, acción y discurso (Chillón 2007). Precisamente por eso este método podría complementarse con análisis discursivos, semióticos o tematológicos, los cuáles nos aportarían datos relevantes sobre los marcos de referencia, la apropiación discursiva, los usos etarios, de género o sociales que se hacen del discurso y por supuesto, con las formas de auto-definirse y definir la realidad cotidiana.

\section{VENTAJAS DEL MÉTODO}

El método biográfico permite a los investigadores sociales situarse en ese punto crucial de convergencia entre el testimonio subjetivo de un individuo ala 
luz de su trayectoria vital, de sus experiencias, de su visión particular, y la plasmación de una vida que es el reflejo de una época. Y por tanto, nos conecta con las normas sociales y los valores esencialmente compartidos con la comunidad de la que el sujeto forma parte (Pujadas 1992, 44). De esta manera, los relatos seleccionados y estudiados nos permitirán comprender y observar de manera activa el fenómeno que nos ocupa y que hemos analizado en profundidad a nivel teórico en los capítulos precedentes. A continuación enumeramos las ventajas a nivel metodológico que nos aporta la realización de historias de vida:

Primero, y como muchos expertos en el tema han apuntado, el método biográfico posibilita en las etapas iniciales de cualquier investigación la formulación o reformulación de hipótesis. Es el caso de Abram Kardiner (1945), por ejemplo, que utiliza las biografías para comprobar si su hipótesis sobre el tipo de personalidad que un tipo de instituciones pueden crear es aproximado en algo a la realidad. Así, debido a la extraordinaria riqueza de matices aportados por los informantes podremos validar las primeras premisas planteadas y cuestionar la correlación causal entre variables.

Segundo, el método nos introduce en profundidad en el universo de las relaciones sociales, de manera que podremos situar a los medios de comunicación dentro del entramado de las estructuras de acogida, los agentes de socialización y la relaciones primarias y secundarias. Es decir, nos proporcionará la comprensión de las variables que explican las mediaciones individuosociedad.

Tercero, como señala Pujadas la utilización de este tipo de fuentes muestra "universales particulares" longitudinalmente, ya que integra esferas sociales y de actividad distinta (familia, trabajo, amistad) y, a la vez, presenta trayectorias concretas y no abstracciones estructurales. Así mismo el autor también señala que existen ventajas a la hora de realizar entrevistas biográficas no solo en la etapa de conclusiones, donde nos sirve como un eficaz "control de los resultados", sino también en la etapa de publicación, ya que "la historia de vida es la mejor "ilustración" posible para que el lector pueda penetrar empáticamente en las características del universo estudiado" (Pujadas 1992, 44-45).

Otro importante investigador que ha remarcado las ventajas del método es Florian Znaniecki, coautor de una de las historias de vida más relevantes titulada El campesino polaco en Europa yen América (1978). El autor considera que una parte esencial de la investigación sociológica es el análisis del significado que las personas otorgan a los procesos. De esta manera, toda realidad social es constituida en la necesaria articulación de actitudes, experiencias, motivaciones, aspiraciones y valoraciones objetivas y subjetivas, colectivas e individuales o, lo que es lo mismo, sociales y personales. El análisis biográfico, por tanto, no tiene porquéser entendido como una simplificación o abstracción excesiva del proceso social, ya que en la investigación de la experiencia de una persona se pueden descubrir elementos estructurales o emociones grupales. 
Esimportante señalar quelas historias de vida no son solo el resultado de una técnica escrupulosa de entrevista en la que se transcriben los elementos propios del habla de cada individuo, sino también, y principalmente, son el resultado de una exhaustiva investigación de carácter científico (Aceves Lozano 1991). Para nosotros, las historias de vida son entendidas como una construcción o proceso interactivo que nos permite tomar parte en el proceso de observación y estudiar el entorno del informante y su manera de autorrelatarse. En su estudio sobre los judíos en América Latina, la investigadora Judit Bokser-Liwerant explica que los archivos orales pueden ilustrar, enriquecer, matizar, completar o reconstruir no solo momentos históricos sino también fenómenos culturales. Además, la historia oral permite enriquecery trascender el plano delainformación sobrelos hechos para acceder al de las representaciones mentales, es decir, el de los valores, cosmovisiones, ideologías, imaginación colectiva (Bokser-Liwerant, 1989).

Tampoco podemos olvidar que la consolidación del movimiento de Historia Oral, como también se suele denominar a la serie de teorías y métodos biográficos, no ha hecho más que tender puentes hacia un proceso convergente en las Ciencias Sociales en los últimos años. Para Pujadas (1992), gracias a este tipo de investigaciones se ha revalorizado el ser humano concreto como sujeto de estudio, por contraste con las excesivas abstracciones y con la deshumanización del cientificismo positivista.

El hecho del que el sujeto emerja como objeto de estudio en sí, no significa que el individuo totalice directamente una sociedad en general, sino que la totaliza a través de la mediación de su contexto social inmediato, de cuyos grupos restringidos forma parte, como ha expresado Franco Ferrarotti (1991). El investigador italiano, muy influido por Karl Marx y Jean-Paul Sartre, considera que todos los individuos totalizan el sistema social, es decir, se apropian de las relaciones sociales, las interiorizan y las transforman en estructuras psicológicas gracias a una actividad que él nombra desestructuración-reestructuración. Ferrarotti reinterpreta el universal singular sartriano ${ }^{2}$ y propone un sistema social capaz de impregnar todos los actos de la persona: todo está reproducido en la historia de vida de cada individuo que sintéticamente introyecta tanto la estructura como la desestructura. (Ferrarotti 1983, 239).

Más que reflejar lo social en sí, "el individuo se lo apropia, lo mediatiza, lo filtra y lo retraduce proyectándolo en otra dimensión, que es la de la subjetividad" (Ferrarroti 1983, 239). Como ha señalado Joan Prat, esta propuesta permite realizar una lectura de la biografía y del sistema social en su totalidad:

Leer una sociedad a través de una biografía es una tentativa legítima y si cada individuo representa la reapropiación de lo universal, social e histórico que lo envuelve, también podemos acercarnos y conocer lo social mediante la especifidad irreductible de una praxis individual (Ferrarroti, 1983, 239).

2 Áquel que reproduce en sí mismo su cultura y su época. 


\section{LAS HISTORIAS DE VIDAY EL ESTUDIO DE LOS EFECTOS}

Aunque pueda pensarse, en una primera instancia, que las historias de vida tienen poco impacto en la comprensión de las estructuras sociales, lo cierto es que las biografías tienen un importante peso en la construcción de los grupos (Anzieu 1986). Es más, la dimensión individual es muy importante en estudios sobre cambio cultural o social, "ya que permite profundizar en el impacto que sobre la vida cotidiana de los individuos tienen las progresivas modificaciones a nivel de estructura" (Pujadas 1992,24).

Asimismo es necesario - tal y como reclama el antropólogo Bronislaw Malinowski (1973)- aportar una visión dinámica de la sociedad, que incorpore la subjetividad y la creatividad humanas y que muestre cómo los individuos responden a los constreñimientos de la estructura social, ensamblándose de forma idiosincrática con los universos sociales y aportando su propia experiencia humana concreta. Y es que resulta esencial encontrar lo general a través de lo particular, lo objetivo a través de lo subjetivo, lo estructural a través de lo histórico, y por ende tender puentes entre la subjetividad expresiva de la conciencia y la objetividad construida de las estructuras sociales.

Como plantea la conocida antropóloga norteamericana Cora Dubois en su conocido estudio The people of Alor (1994), es necesario utilizar los materiales biográficos para tratar la variable individual (psicológica) en relación a las variables culturales (instituciones primarias y secundarias) entrelas que nosotros podríamos incluir a los medios de comunicación. La realización de historias de vida permitirá, por tanto, profundizar o comprender mejor fenómeno concretos, tales como son la recepción mediática o la participación virtual de los sujetos y los grupos.

En los últimos años, diversos investigadores se han centrado en cómo diferentes colectivos utilizan los significados que circulan en los medios para dar sentido a sus vidas y a sus categorizaciones o percepciones de clase, etnia u otras formas de identificación. Tal y como han sugerido Peter L. Lunt y Sonia M. Livingstone (1992), el consumo penetra en el significado de la experiencia psicológica afectando a la construcción de identidades o la formación de relaciones sociales. A este respecto, destacan las aportaciones de David Morley (1986), John Fiske y Jonh Hartley (1978) o James Lull (1988), pioneros en los estudios etnográficos sobre los usos de la televisión. La característica común de estos primeros trabajos es que toman como marco de partida la familia y el hogar como lugar donde surgen relaciones de dominio, conflicto, toma de decisiones y establecimiento de reglas (Goodman 1983).

Entre las contribuciones más actuales podemos mencionar las de Miguel Ángel Aguilar, Rosalía Winocur (2005) o Néstor García Canclini (2005), investigadores que han estudiado los contextos de recepción y los procesos de apropiación por parte de los receptores, aunando los aportes de la antropo- 
logía y los estudios de comunicación. Como ha subrayado Dolors Comas d'Argemir, este tipo de enfoques tratan de establecer mediante la etnografía el modo en que los espectadores interpretan los mensajes y la manera en que utilizan las representaciones para asumir las ideologías enraizadas en los textos, para crear identidades y para imaginar otras realidades $(2000,184)$.

En una línea similar, podemos señalar las aportaciones de Larry Nathan Stelitz (2002), que ha realizado estudios etnográficos sobre la recepción mediática y la configuración de identificaciones sociales. Valga señalar que el investigador combina el uso de historias de vida, grupos de discusión y observación participante para explorar el modo en los sujetos apropian y refiguran los contenidos representados. También Amit Kama (2005; 2002) ha centrado sus investigaciones en la influencia de los medios en la construcción de la identidad gay, en concreto, y utiliza el método biográfico como vía para entender el modo en que los sujetos se autoperciben y se sienten juzgados y evaluados por los otros. Este tipo de enfoques son muy enriquecedores y ponen de evidencia las ventajas de tender puentes entres disciplinas para poder abordar fenómenos complejos que afectan tanto a lo individual como a lo colectivo.

Como menciona Luciano H. Elizalde, en Los jóvenes y sus relaciones cotidianas con los medios (1998), los estudios de los efectos en líneas generales siguen manteniendo una hipótesis fuerte, que afirma y defiende la causalidad de los medios y de sus productos en la manera de pensar y de comportarse de las personas. Sin embargo, algunas de estas hipótesis no terminan de explicar muy bien cómo se da este proceso de causa-efecto. En este sentido, consideramos que las historias de vida pueden adentrarse con muy buenos resultados en estos procesos a un nivel psico-social. Además, la mayoría de los estudios de efectos utilizan estrategias metodológicas experimentales que se centran en las audiencias generales y en los efectos estructurales, olvidando a veces lo que sucede en la realidad cotidiana entre los sujetos individuales y los medios. Como bien anota J. Habermas:

\footnotetext{
Los estudiosos sobre los medios de comunicación dan información en cierto modo fiable acerca del marco institucional y de la estructura, así como de la forma de trabajo, la configuración de los programas y la utilización de los medios; pero las afirmaciones sobre los efectos de los medios siguen siendo inseguras, incluso una generación después de Lazarsfeld $(1998,458)$.
}

En este sentido, consideramos que el método no puede aportar resultados universales o falseados sino aportar conclusiones interpretativas que nos permitan describir y observar los diferentes procesos que se dan en la recepción mediática o en la participación virtual. Recordemos aquí la propuesta semiótica de la cultura propuesta por el antropólogo Cliford Geertz (1988). Para el autor, desde una matriz semiótica la antropología trasciende la pretensión de 
constituir un"ciencia experimental en busca deleyes" para elaborar una ciencia interpretativa o antropología interpretativa en busca de significaciones. Para ello consideramos que los estudios de comunicación más que centrarse en el proceso de decodificación deberían focalizar en el análisis de las variantes psico-sociales. Esto permitiría profundizar en el entramado de las relaciones sociales objetivas y subjetivas que los individuos realizan al entrar en contacto con las narrativas mediáticas, sea en la recepción clásica como en la participación virtual.

Entre los estudios más recientes sobre los efectos de los medios que utilizan historias de vida como metodología principal hemos las aportaciones de Víctor F. Sampedro (2004) sobre los jóvenes de las favelas brasileñas también dan buena cuenta de las posibilidades de este método. El autor estudia, junto su equipo de investigadores, las relaciones de los individuos y los grupos con las identidades representadas en los medios de comunicación mediante la realización de historias de vidas con técnicas de observación participante.

\section{CONCLUSIONES}

En definitiva, el uso de historias de vida puede aportar elementos comprensivos fundamentales en investigaciones sobre recepción mediática y participación virtual. Este enfoque no solo puede servir para validar hipótesis en una primera etapa de una investigación, sino también para el desarrollo de teorías acerca de los muchos procesos psico-sociales que se dan como efecto del consumo mediático. Así las historias de vida pueden ilustrar aportes, complementar otros instrumentos metodológicos, como la encuesta, la observación participante o los grupos de discusión. A través del análisis de una muestra significativa, coherente con el objeto de estudio y con los objetivos planteados, se puede llegar a conclusiones muy interesantes. No obstante, valga reiterar que esta metodología nunca podrá mostrar valores universales o generalizar tendencias, ya que profundiza en la variable individual (la vivencia histórico-subjetiva del informante o entrevistado). Pero no por ello estos elementos son menos valiosos. Al fin y al cabo, los fenómenos o procesos de recepción o participación virtual son vividos por los individuos en primera persona pero en estricta conexión con su grupo de referencia, su marco cultural o contexto socio-histórico. De manera, que los resultados traerán a colación necesariamente pautas de comportamiento social, formas culturales propias de la época o el grupo social estudiado y nos dirán mucho del fenómeno en sí. 


\section{REFERENCIAS}

Aceves Lozano, JorgeE.(1991). Historia orale Historias devida. Teoría, métodosytécnicas. Una biografía comentada. Centro de Investigaciones y Estudios Superiores en Antropología Social.

Aguilar, Miguel Ángel y Winocur, Rosalía (2005). Ciudad y medios de comunicación en García Canclini, Néstor (coord.) La antropología urbana en México. México: Fondo de Cultura Económica, 197-220.

Anderson, Rufus (1825). Memoir of Catherine Brown a Christian Indian of the Cherokee Nation. Nueva York, NY: J. P. Haven.

Anzieu, Didier (1986). El grupo y el inconsciente. Madrid: Biblioteca Nueva.

Augé, Marc (1996). El sentido de los otros. Barcelona: Paidós.

Bajtín, Mijaíl (1975). Teoría y estética de la novela. Madrid: Editorial Taurus.

Bokser-Liwerant, Judit (1989). Reencontrando identidad. Apuntes metodológicos para el estudio de los judíos en América Latina, Revista Americana de Ciencias Sociales, n¹3, enero-abril. México: Instituto Mora, 207-215.

Booth, Charles (1903). Life and Labour of the People of London. Londres: Macmillan.

Buhler, Charlotte (1930). Personality Types Based on Experiments with Children. Informe presentado en el IX Congreso Internacional de Psicología. New Haven, CT.

Chillón, Albert (2007). Hacer los hechos. Un ensayo de fenomenología de los 'hechos sociales'. Ars Brevis, 13, Universitat Ramon Llull, Barcelona, 27-50.

Dubois, Cora (1994). The people of Alor. Minneapolis University of Minnesota Press.

Elizalde, Luciano H. (1999). Los jóvenes y sus relaciones cotidianas con los medios. Una aproximación teórica y metodológica al estudio de los efectos. Cuadernos australes de comunicación. Facultad de Ciencias de la Información. Buenos Aires: Universidad Austral.

Ferraroti, Franco (1990). Time, Memory and Society. Westport, CT: Greenwood Press.

Ferrarotti, Franco. (1983). Histoire et histoires de vie. En La méthode biographique dans les sciences sociales. Paris: Librairie des Méridiens.

Ferrarotti, Franco. (1991). La historia y lo cotidiano. Buenos Aires: CEAL.

Fiske, John y Hartley, John (1978). Reading TV. Londres: Methuen. 
García Canclini, Néstor(2006).Diferentes, desigualesy desconectados. Barcelona: Gedisa.

Geertz, Clifford (1981). La interpretación de las culturas. Barcelona: Gedisa.

Goodman, Irene F. (1983). Television's Role in Family Interaction: A Family Systems Perspective. Journal of Family Issues, 4, junio, 405-424.

Habermas, Jürgen (1988). La lógica de las ciencias sociales. Madrid: Editorial Tecnos.

Kama, Amit (2002). The Quest of Inclusion: Jewish-Israeli Gay Men's Perception of Gays in the Media. Feminist Media Studies, 2(2), julio, 195-212.

Kama, Amit (2005). A Unrelating Mental Press: Israeli Gay Men’s Ontological Duality And its Discontent. The Journal of Men's Studies, 13(2), enero, 168-184.

Kardiner, Abraham (1945). El individuo y su sociedad. México: Fondo de Cultura Económica.

Kardiner, Abraham (1955). Las fronteras psicológicas de la sociedad. México: Fondo de Cultura Económica.

Kluckhohn, Clyde (1945). The Personal Document in Anthropological Science Gottschalk, Louis; Kluckhohn, Clyde y Angell, Robert (eds.) The Use of Personal Documents in History, Anthropology and Sociology. Nueva York, NY: Social Science Research Council, 77-173.

Le Play, P. G. Frédéric (1855). Les ouvriers européens. Études sur les travaux, la vie et la condition moral des populations ouvrières de l'Europe. Précédées d'un exposé de la méthode d’observation. París: Imprimerie Impériale.

Lévi-Strauss, Claude (2008). ¿La antropología en peligro de muerte? El Correo de la Unesco, 5, 39-46.

Llull, James (1988). World Families Watch Television. Newbury Park, CA: Sage.

Lunt, Peter K. y Livingstone, Sonia M. (1992). Mass Consumption and Personal Identity: Everyday Economic Experience. Buckingham: Open University Press.

Malinowski, Bronislaw (2000). Los argonautas del Pacífico occidental. Barcelona: Península.

Misch, George (1951). A History of Autobiography in Antiquity. Cambridge, MA: Harvard University Press.

Mitchell, G. Duncan (1988). Historia de la sociología. Barcelona: Editorial Labor.

Morley, David (1986). Family Television: Cultural Power and Domestic Leisure. Londres: Comedia.

Prat, Joan (2007). Los sentidos de la vida. La construcción del sujeto, modelos del yo e identidad. Bellaterra: Ediciones Bellaterra. 
Pujadas, Juan José (1992). El método biográfico. El uso de las historias de vida en ciencias sociales. Madrid: Centro de Investigaciones Sociológicas.

Said, Edward (1994). Cultura e Imperialismo. Barcelona: Anagrama.

Sampedro, Víctor F. (2004). Identidades mediáticas e identificaciones mediatizadas. Visibilidad y reconocimiento identitario en los medios de comunicación. Revista CIDOB d'Afers Internacionals, 66-67, Centro de Estudios y Documentación Internacionales de Barcelona (CIDOB), 135-149.

Strelitz, Larry N. (2002). Media Consumption and Identity Formation: The Case of 'Homeland' Viewers. Media, Culture and Society, 24(4), julio, 459-480.

Szczepanski, Jan (1978). El método biográfico. Papers. Revista de Sociologia, 10, Universitat Autònoma de Barcelona, Bellaterra, 231-259.

Thomas, William y Znaniecki, Florian (2004). El campesino polaco en Europa y en América. Madrid: Centro de Investigaciones Sociológicas.

Thompson, Paul(1988).La voz delpasado: La historia oral. Valencia:Institución Alfonso el Magnánimo. 